\title{
AVALIAÇÃO DE MATERIAIS EDUCATIVOS DIRECIONADOS PARA O DESENVOLVIMENTO NEUROPSICOMOTOR DA CRIANÇA
}

\section{Evaluation of educational materials targeted at the psychomotor development of the child Evaluación de materiales educativos dirigidos al desarrollo neuropsicomotor del niño}

\author{
Hitallo Lima Silva \\ Centro Universitário Estácio de Ceará - Fortaleza (CE) - Brasil. \\ Flávia Helena Germano Bezerra \\ Centro Universitário Estácio de Ceará - Fortaleza (CE) - Brasil. \\ Ismênia de Carvalho Brasileiro \\ Centro Universitário Estácio de Ceará - Fortaleza (CE) - Brasil.
}

\section{RESUMO}

Objetivo: Avaliar na literatura materiais educativos desenvolvidos de modo impresso ou on-line que sejam direcionados para promoção, prevenção ou intervenção sobre o desenvolvimento neuropsicomotor da criança. Métodos: Tratou-se de um estudo de revisão integrativa, em que foram pesquisados artigos nos idiomas português e inglês nas bases de dados biblioteca virtual Scientific Electronic Library Online (SciELO), Google Acadêmico (GA), Biblioteca Regional de Medicina (Bireme), Physiotherapy evidence database (Pedro) e PubMed, entre janeiro e março de 2017, com os termos cartilha (booklet), criança (children) e desenvolvimento motor (motor development). Após leitura de títulos e resumos, e tomando como base os princípios de uma revisão integrativa, foram excluídos 7224 estudos por não contemplarem os critérios solicitados, permanecendo uma amostra de 8 artigos. Resultados: Os estudos que realizaram a avaliação e análise de cartilhas educativas mostraram que esses materiais têm respostas positivas como um instrumento adequado para auxiliar pais, famílias, estudantes e profissionais de saúde nas atividades de educação em saúde. Observou-se que a intervenção precoce realizada pelos familiares teve resultados positivos relacionados à evolução da criança, afetando seu desenvolvimento linguístico e motor nos casos estudados. Conclusão: As evidências científicas apontam que os materiais educativos em saúde elaborados para orientação de pais e profissionais de saúde sobre a estimulação do desenvolvimento infantil são considerados claros, objetivos e eficientes.

Descritores: Recursos Materiais em Saúde; Criança; Desenvolvimento Infantil; Promoção da Saúde.

\section{ABSTRACT}

Objective: To evaluate in the literature the educational materials developed on printed or online versions that are targeted at the promotion, prevention or intervention regarding the psychomotor development of the child. Methods: The study consisted in an integrative review, where articles were searched in Portuguese and English languages, in the databases: Scientific Electronic Library Online (SciELO), Google academic, Regional Library of Medicine (Bireme), Physiotherapy evidence database (Pedro) e PubMed, between January and March 2017, using booklet (cartilha), children (criança) and motor development (desenvolvimento motor) as search terms. After reading the titles and abstracts, and based on the principles of an integrative review, 7,224 studies were excluded for not meeting the requested criteria, remaining a sample of 8 articles. Results: The studies that conducted the evaluation and analysis of educational booklets showed that these materials led to positive responses as an appropriate instrument to assist parents, families, students and health professionals in health education activities. It was observed that the early intervention performed by family members had positive results concerning the evolution of the child, influencing on their language and motor development in the cases studied. Conclusion: Scientific evidence points out that health education materials designed for guidance of parents and health professionals on the stimulation of child development are considered clear, objective and efficient.

Descriptors: Material Resources in Health; Child; Child Development; Health Promotion. 


\section{RESUMEN}

Objetivo: Evaluar los materiales educativos desarrollados de modo impreso u on-line en la literatura que sean dirigidos a la promoción, prevención o intervención del desarrollo neuropsicomotor del niño. Métodos: Se trató de un estudio de revisión integrativa en el cual fueron investigados artículos en los idiomas portugués y inglés de las bases de datos biblioteca virtual Scientific Electronic Library Online (SciELO), Google Académico (GA), Biblioteca Regional de Medicina (Bireme), Physiotherapy evidence database (Pedro) y PubMed entre enero y marzo de 2017 con los términos cartilla (booklet), niño (children) y desarrollo motor (motor development). Tras la lectura de los títulos y resúmenes y basado en los principios de una revisión integrativa se excluyeron 7224 estudios por el no cumplimiento a los criterios determinados quedándose una muestra de 8 artículos. Resultados: Los estudios que realizaron la evaluación y el análisis de las cartillas educativas mostraron que eses materiales tienen respuestas positivas de un instrumento adecuado para ayudar a los padres, las familias, los estudiantes y profesionales sanitarios en las actividades de educación en salud. Se observó que la intervención precoz realizada por parte de los familiares tuvo resultados positivos relacionados con la evolución del niño afectando su desarrollo lingüístico y motor de los casos estudiados. Conclusión: Las evidencias científicas señalan que los materiales educativos en salud elaborados para orientar a los padres y profesionales sanitarios sobre la estimulación del desarrollo infantil son considerados claros, objetivos y eficientes.

Descriptores: Recursos Materiales en Salud; Niño; Desarrollo Infantil; Promoción de la Salud.

\section{INTRODUÇÃO}

O desenvolvimento motor (DM) infantil abrange os diferentes componentes da motricidade, como a coordenação, o equilíbrio e o esquema corporal ${ }^{(1)}$. É um processo sequencial relacionado à idade cronológica trazido pela interação entre as habilidades de realizar as tarefas, o ambiente em que a criança vive e a biologia do indivíduo, podendo ser influenciado pelas mudanças sociais, intelectuais e emocionais ${ }^{(2)}$.

Com o intuito de promover o desenvolvimento pleno infantil e de prevenir eventuais atrasos oriundo de uma diversidade de fatores pré, peri ou pós-natais, têm sido difundidos um arsenal de possibilidades, dentre elas, o uso crescente de materiais educativos como ferramenta de educação em saúde que gera possibilidades de ensino-aprendizagem por meio da interação entre familiares e criança, gerando prognósticos $\operatorname{positivos}^{(3)}$.

Esses materiais escritos são instrumentos que facilitam o processo educativo, pois permitem ao leitor reforçar informações e discussões orais oriundas de outros meios, servindo como guia de orientações para decisões mais assertivas ${ }^{(4)}$.

Assim, cartilhas e folhetos informativos são descritos como parte da educação ou promoção da saúde, tendo como objetivos a prevenção, tratamento e cumprimento de tarefas. Alguns autores relatam, entretanto, que quando mal confeccionadas dificultam o entendimento e a compreensão dos usuários ${ }^{(5)}$. O material educativo deve ser ter linguagem acessível, uma vez que quanto menor o nível de leitura, mais provável que seja lido por grande parte do público-alvo ${ }^{(6)}$.

Além disso, dado o avanço tecnológico, observa-se cada vez mais a possibilidade de disseminação de informações em saúde em mídias digitais, uma vez que panfletos e folhetos escritos são limitados em relação ao acesso ${ }^{(7)}$.

Mesmo com um arsenal de documentos disponível impresso ou digital, percebe-se uma lacuna no conhecimento sobre a quantidade e em especial a qualidade de materiais educativos para crianças com agravos ou riscos para o desenvolvimento neuropsicomotor.

Diante dessas premissas, questiona-se: Como têm sido desenvolvidos e difundidos materiais educativos para as crianças que possam vir a apresentar algum agravo no curso do seu desenvolvimento infantil?

Dessa forma, o estudo teve como objetivo avaliar na literatura materiais educativos desenvolvidos de modo impresso ou on-line que sejam direcionados para promoção, prevenção ou intervenção sobre o desenvolvimento neuropsicomotor da criança.

\section{MÉTODOS}

Tratou-se de um estudo de revisão integrativa, em que se buscou na literatura vigente avaliar materiais educativos desenvolvidos para promoção, prevenção ou intervenção na saúde que abordassem o desenvolvimento neuropsicomotor da criança.

Após definição do objetivo específico, foi formulada a hipótese de que os materiais educativos desenvolvidos sobre desenvolvimento neuropsicomotor são escassos e de pouca profundidade quanto à clareza e riqueza de dados aos familiares, cuidadores e comunidade acerca do desenvolvimento infantil, particularmente no que diz respeito ao eixo neuropsicomotor.

A partir de então, iniciou-se a busca para identificação e coleta do máximo de pesquisas primárias relevantes dentro dos critérios de inclusão e exclusão previamente estabelecidos. Foram incluídos artigos que utilizassem e/ou avaliassem cartilhas, nos últimos 10 anos, publicados em português e inglês, e disponíveis em formato de texto completo. Excluíram-se relatos 
de experiências, revisões, cartas e artigos que, embora abordassem sobre o assunto proposto nos títulos e nos resumos, não especificassem no texto o material, ou metodologia, que configurasse material educativo.

Para tanto, foram pesquisados artigos nos idiomas português e inglês nas bases de dados biblioteca virtual Scientific Electronic Library Online (SciELO), Google Acadêmico (GA), Biblioteca Regional de Medicina (Bireme), Physiotherapy evidence database (Pedro) e PubMed, entre janeiro e março de 2017, com os termos cartilha (booklet), criança (children) e desenvolvimento motor (motor development).

Inicialmente foram identificados 7236 artigos [GA $(n=7060)$; SciELO $(n=94)$; Bireme $(n=24)$; Pubmed $(n=51)$; Pedro $(\mathrm{n}=7)$ ], mas, após leitura do título e resumo, e tomando como base os princípios de uma revisão integrativa que preconizam a avaliação dos critérios e métodos empregados no desenvolvimento dos estudos selecionados para determinar sua validade metodológica, foram excluídos 7224 por não contemplarem os critérios solicitados, permanecendo uma amostra de 8 artigos. O fluxograma mostra a extração dos estudos incluídos na pesquisa (Figura 1).

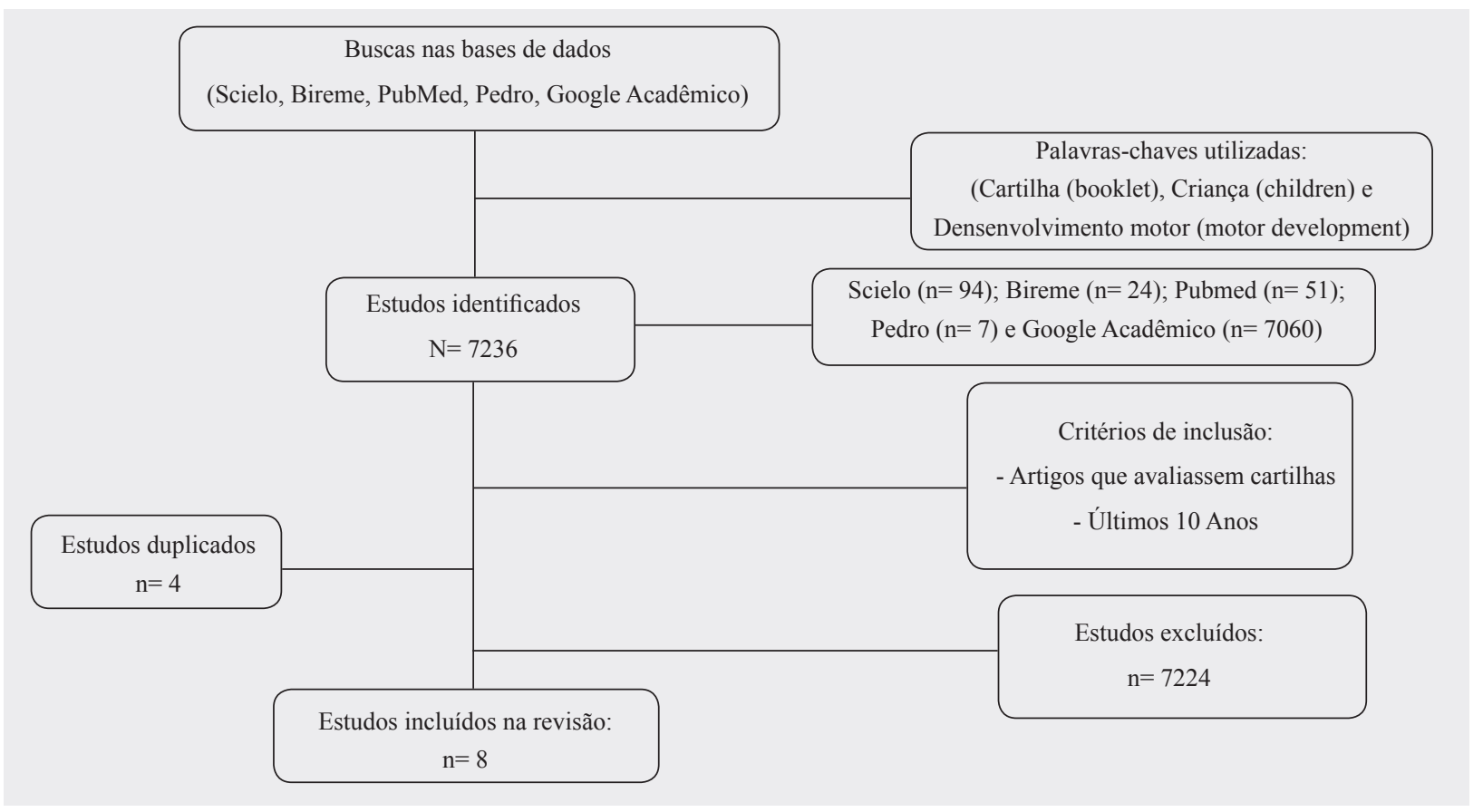

Figura 1 - Estratégia de busca e seleção dos estudos.

\section{RESULTADOS}

Dos artigos encontrados, cinco deles avaliaram ou aplicaram cartilhas educativas e expressaram os resultados apresentados pelo público-alvo. Outros três estudos aplicaram programas terapêuticos em maneira direta com a criança e em formato de folhetos educativos (Tabela I). 
Tabela I - Análise descritiva dos artigos incluídos no estudo.

\begin{tabular}{|c|c|c|c|c|}
\hline Autor, Ano & $\begin{array}{c}\text { Material } \\
\text { desenvolvido }\end{array}$ & Objetivos & Principais resultados & Conclusão \\
\hline $\begin{array}{l}\text { Grippo et al., } \\
2008^{(8)}\end{array}$ & $\begin{array}{l}\text { Avaliação de cartilha } \\
\text { educativa. }\end{array}$ & $\begin{array}{l}\text { Analisar a percepção dos } \\
\text { familiares sobre os temas } \\
\text { discutidos na cartilha e identificar } \\
\text { a aceitação da cartilha pela } \\
\text { população. }\end{array}$ & $\begin{array}{l}\text { Observou-se uma predominância } \\
\text { de mães com ensino fundamental } \\
\text { incompleto que relataram } \\
\text { compreensão e interesse pela } \\
\text { cartilha. }\end{array}$ & $\begin{array}{llr}\text { Existem } & \text { evidências } & \text { de } \\
\text { efetividade da cartilha } & \text { como } \\
\text { instrumento } & \text { promotor } & \text { de } \\
\text { habilidades. } & & \\
\text { her } & & \end{array}$ \\
\hline $\begin{array}{l}\text { Guimarães et al., } \\
2015^{(9)} \text {. }\end{array}$ & $\begin{array}{l}\text { Avaliação de cartilha } \\
\text { educativa. }\end{array}$ & $\begin{array}{l}\text { Avaliar a clareza e objetividade } \\
\text { de uma cartilha com explicações } \\
\text { detalhadas e ilustrações } \\
\text { acerca do desenvolvimento } \\
\text { neuropsicomotor de crianças de } \\
0 \text { a } 6 \text { anos. }\end{array}$ & $\begin{array}{l}\text { A grande maioria dos participantes } \\
\text { da pesquisa avaliou a cartilha } \\
\text { como boa ou ótima }(96 \%) \text {. }\end{array}$ & $\begin{array}{l}\text { A cartilha mostrou-se como uma } \\
\text { medida prática e de baixo custo } \\
\text { que pode auxiliar na estimulação } \\
\text { do desenvolvimento infantil. }\end{array}$ \\
\hline $\begin{array}{l}\text { Ichini et al } \\
2016^{(10)}\end{array}$ & $\begin{array}{l}\text { Intervenção precoce } \\
\text { no desenvolvimento } \\
\text { infantil. }\end{array}$ & $\begin{array}{l}\text { Analisare compararas percepções } \\
\text { de pais e dos terapeutas sobre os } \\
\text { efeitos da intervenção precoce no } \\
\text { desenvolvimento de seus filhos e } \\
\text { no cuidado aos mesmos. }\end{array}$ & $\begin{array}{l}\text { Dos nove sujeitos analisados, sete } \\
\text { apresentaram percepção parental } \\
\text { e terapêutica semelhante para } \\
\text { as questões do desenvolvimento } \\
\text { abordadas e em dois casos houve } \\
\text { divergência entre a visão dos } \\
\text { familiares e dos terapeutas acerca } \\
\text { do desenvolvimento das crianças. }\end{array}$ & $\begin{array}{l}\text { A percepção das terapeutas } \\
\text { e pais demonstrou efeitos } \\
\text { positivos da intervenção precoce } \\
\text { interdisciplinar, manifestada na } \\
\text { concordância de pontos de vista } \\
\text { em relação aos sete dos nove } \\
\text { sujeitos analisados. }\end{array}$ \\
\hline $\begin{array}{l}\text { Fonseca et al., } \\
2007^{(11)}\end{array}$ & Cartilha educativa. & $\begin{array}{l}\text { Descrever o processo } \quad \text { de } \\
\text { divulgação e repercussão da } \\
\text { cartilha educativa nos meios de } \\
\text { comunicação. }\end{array}$ & $\begin{array}{l}\text { Apreendeu-se que a cartilha teve } \\
\text { excelente aceitação, pois atende às } \\
\text { necessidades da clientela, ajuda na } \\
\text { autoconfiança, possibilita o retorno } \\
\text { do conhecimento produzido para a } \\
\text { sociedade. }\end{array}$ & $\begin{array}{l}\text { A cartilha constitui instrumento } \\
\text { adequado para auxiliar } \\
\text { pais, família, estudantes e } \\
\text { profissionais de saúde nas } \\
\text { atividades de educação em } \\
\text { saúde. }\end{array}$ \\
\hline $\begin{array}{l}\text { Ferecini et al } \\
2009^{(12)}\end{array}$ & $\begin{array}{l}\text { Utilização de cartilha } \\
\text { educativa. }\end{array}$ & $\begin{array}{l}\text { Analisar a percepção de mães de } \\
\text { prematuros sobre a vivencia em } \\
\text { um Programa de Educação em } \\
\text { Saúde. }\end{array}$ & $\begin{array}{l}\text { Apreenderam-se núcleos } \\
\text { temáticos: o aprendizado } \\
\text { proporcionado pelo Programa; } \\
\text { a criação de possibilidades de } \\
\text { socializar o conhecimento com a } \\
\text { família e o Programa como espaço } \\
\text { para descontração e escuta. }\end{array}$ & $\begin{array}{l}\text { Recomenda-se que programas } \\
\text { educativos dessa natureza e } \\
\text { ampliados com a participação } \\
\text { de outros membros da família do } \\
\text { prematuro sejam implantados } \\
\text { em outras unidades neonatais. }\end{array}$ \\
\hline $\begin{array}{l}\text { Fracolli } \\
\text { et al., 2010 }\end{array}$ & $\begin{array}{l}\text { Avaliação de cartilha } \\
\text { educativa. }\end{array}$ & $\begin{array}{l}\text { Avaliar a utilização da cartilha } \\
\text { pelas famílias. }\end{array}$ & $\begin{array}{l}\text { Os resultados mostraram que a } \\
\text { cartilha foi muito utilizada pelas } \\
\text { famílias. Por ocasião da pesquisa } \\
72,4 \% \text { das famílias ainda possuíam } \\
\text { a cartilha e } 81 \% \text { conversavam } \\
\text { sobre o conteúdo da cartilha com } \\
\text { profissionais da saúde da equipe de } \\
\text { saúde da família. }\end{array}$ & $\begin{array}{l}\text { Concluiu-se que a cartilha } \\
\text { foi um instrumento adequado } \\
\text { de educação em saúde pois } \\
\text { viabilizou o diálogo sobre o } \\
\text { desenvolvimento infantil entre } \\
\text { famílias e profissionais de } \\
\text { saúde. }\end{array}$ \\
\hline Couto et al., 2012 (14). & $\begin{array}{l}\text { Suporte materno no } \\
\text { domicílio. }\end{array}$ & $\begin{array}{l}\text { Identificar o suporte materno, } \\
\text { no domicílio, para o cuidado } \\
\text { do recém-nascido prematuro } \\
\text { egresso de unidade neonatal. }\end{array}$ & $\begin{array}{l}\text { Os participantes valorizaram a } \\
\text { Cartilha de orientação oferecida } \\
\text { pelo serviço; e destacaram a } \\
\text { relevância do apoio social para o } \\
\text { cuidado do bebê, no domicílio. }\end{array}$ & $\begin{array}{l}\text { Recomenda-se a inserção } \\
\text { do familiar no plano de } \\
\text { enfermagem para a alta do } \\
\text { prematuro na Unidade Neonatal. }\end{array}$ \\
\hline Farris et al $2013^{(15)}$. & $\begin{array}{l}\text { Programa } r \text { de } \\
\text { intervenção através } \\
\text { de folheto. }\end{array}$ & $\begin{array}{l}\text { Avaliar os efeitos secundários de } \\
\text { um programa de intervenção de } \\
\text { treinamento de pais. }\end{array}$ & $\begin{array}{l}\text { Os achados indicaram que todos } \\
\text { os níveis de intervenção foram } \\
\text { associados com aumentos no bem- } \\
\text { estar materno para participantes } \\
\text { com crianças tipicamente em } \\
\text { desenvolvimento. }\end{array}$ & $\begin{array}{l}\text { A identificação precoce de } \\
\text { mães enfrentando problemas } \\
\text { de comportamento infantil e a } \\
\text { disseminação de programas de } \\
\text { intervenção preventiva com boa } \\
\text { relação custo-benefício pode ter } \\
\text { resultados significativos. }\end{array}$ \\
\hline
\end{tabular}




\section{DISCUSSÃO}

O uso de materiais educativos em saúde apontou resultados positivos no que diz respeito ao auxílio dos pais quanto às abordagens terapêuticas a serem desenvolvidas em ambientes domiciliares e hospitalares para melhorar, ou reverter, quadros de atrasos no desenvolvimento infantil.

Os estudos que realizaram a avaliação e análises de cartilhas educativas mostraram que os materiais educativos têm respostas positivas como um instrumento adequado para auxiliar pais, famílias, estudantes e profissionais de saúde nas atividades de educação em saúde. Foi observado que há uma predominância de mães com ensino fundamental incompleto que relataram compreensão e interesse pelos conteúdos apresentados nos materiais ${ }^{(8)}$.

O artigo que descreveu a análise de uma cartilha por avaliadores compostos por pais, professores e fisioterapeutas, mostrou que $96 \%$ dos participantes avaliaram como bom ou ótimo o material para favorecer a estimulação do desenvolvimento motor infantil. Todos os pais e professores concluíram que aprenderam algo novo ao ler o material e quase metade dos fisioterapeutas também relataram esse fato( ${ }^{(9)}$.

A importância dos cuidados no domicílio foi um item mencionado nos estudos. Observou-se que a intervenção precoce realizada pelos familiares teve resultados positivos relacionados à evolução da criança, afetando seu desenvolvimento linguístico e motor nos casos estudados ${ }^{(10)}$.

Um estudo que disponibilizou o material pela internet, meio de ampla rede de comunicação, facilitou a circulação do conteúdo proposto para todas as regiões do país, alcançando um maior número de leitores ${ }^{(11)}$.

Os programas de educação em saúde aplicados para mães de bebês prematuros, a partir de uma série de atividades em grupos, discutindo sobre temas relacionados aos cuidados com o bebê, evidenciaram contribuição para o cuidado dos filhos tanto no hospital como em domicílio ${ }^{(12)}$.

A cartilha em formato on-line, ou digital, apresentou-se como importante recurso do acesso dos leitores, não sendo ajustado para população que frequenta unidades públicas de saúde e usuários do SUS, que geralmente são pessoas que não têm acesso ao meio digital, tornando necessário o uso de materiais impresso ${ }^{(16)}$.

Essa é uma população de risco e vulnerável a ocorrência de agravos no desenvolvimento da criança, que pode ser influenciado pelo ambiente, e agravado por fatores como a questão financeira dos pais, acesso à saúde, educação, alimentação e habitação. Crianças que são baixa renda têm maiores chances de apresentarem suspeitas de atraso no desenvolvimento do que as crianças de famílias com aspectos financeiros piores ${ }^{(17)}$.

Em ambiente hospitalar, a que muitas vezes as crianças são submetidas por diversos fatores, também é preciso a interação dos profissionais de saúde com os pais para educá-los, evitando atrasos futuros no crescimento dos seus bebês. Portanto, os profissionais devem utilizar-se de recursos educacionais como estratégia terapêutica efetiva para consolidação do aprendizado das famílias nesse espaço, com consequente benefício para criança ${ }^{(18)}$.

A precariedade de estudos e pesquisas publicadas relacionados ao tema desse estudo evidencia a necessidade de profissionais estarem desenvolvendo estudos na área que gerem resultados positivos na saúde da população.

É preciso socializar o conhecimento produzido, e as tecnologias atuais de informação são necessárias e capazes de difundir tal conhecimento. Com o avanço tecnológico, os profissionais devem utilizar mais recursos em prol da educação em promoção da saúde, desenvolvendo ferramentas, como aplicativos, jogos, sites e cartilhas on-line, dentre outros.

\section{CONCLUSÃO}

As evidências científicas apontam que os materiais educativos em saúde elaborados para orientação de pais e profissionais de saúde sobre a estimulação do desenvolvimento infantil são considerados claros, objetivos e eficientes.

\section{REFERÊNCIAS}

1. Medina-Papst J, Marques I. Avaliação do desenvolvimento motor de crianças com dificuldades de aprendizagem. Rev Bras Cineantropom Desempenho Hum. 2010;12(1):36-42.

2. Rosa F Neto, Santos APM, Xavier RFC, Asmaro KN. A Importância da avaliação motora em escolares: análise da confiabilidade da Escala de Desenvolvimento Motor. Rev Bras Cineantropom Desempenho Hum. 2010;12(6):422-7.

3. Freitas AAS, Cabral IE. O cuidado à pessoa traqueostomizada: análise de um folheto educativo. Esc Anna Nery Rev Enferm 2008;12(1):84-9.

4. Moreira MF, Nóbrega MML, Silva MIT. Comunicação escrita: contribuição para a elaboração de material educativo em saúde. Rev Bras Enferm. 2003;56(2):184-8. 
5. Protheroe J, Estacio EV, Saidy-Khan S. Patient information materials in general practices and promotion of health literacy: an observational study of their effectiveness. Br J Gen Pract. 2015;65(632):e192-7.

6. Moult B, Franck LS, Brady H. Ensuring Quality Information for patients: development and preliminary validation of a new instrument to improve the quality of written health care information. Health Expect. 2004;7(2):165-75.

7. Armstrong AW, Idriss NZ, Kim RH. Effects of video-based, online education on behavioral and knowledge outcomes in sunscreen use: A randomized controlled trial. Patient Educ Couns. 2011;83(2):273-7.

8. Grippo MLVS, Fracolli LA. Avaliação de uma cartilha educativa de promoção ao cuidado da criança a partir da percepção da família sobre tema de saúde e cidadania. Rev Esc Enferm USP 2008;42(3):430-6.

9. Guimarães FAB, Assis CD, Vieira MEB, Formiga CKMR. Avaliação de material didático elaborado para orientação de cuidadores e professores de creches sobre o desenvolvimento infantil. Rev Bras Crescimento Desenvolv Hum. 2015;25(1):27-40.

10. Pichine FS, Rodrigues NGS, Ambrós TMB, Souza APR. Percepção da família e do terapeuta sobre a evolução de crianças em uma abordagem interdisciplinar de intervenção precoce. Rev CEFAC 2016;18(1):55-66.

11. Fonseca LMM, Leite AM, Vasconcelos MGL, Castral TC, Scochi CGS. Cartilha educativa on line sobre os cuidados com o bebê prétermo: aceitação dos usuários. Ciênc Cuid Saúde. 2007;6(2):238-44.

12. Ferecini GM, Fonseca LMM, Leite AM, Daré MF, Assis CS, Scochi CGS. Percepções de mães de prematuros acerca da vivencia em um programa educativo. Acta Paul Enferm. 2009;22(3):250-6.

13. Fracolli LA, Chiesa AM. A percepção das famílias sobre a cartilha "toda hora é hora de cuidar". Mundo Saúde. 2010;34(1):36-42.

14. Couto ff, Praça NS. Recém-nascido prematuro: suporte materno domiciliar para o cuidado. Rev Bras Enferm. 2012;65(1): 19-26.

15. Farris JR, Bert SSC, Nicholson JS, Glass K, Borkowski JG. Effective Intervention Programming: Improving Maternal Adjustment Through Parent Education. Adm Policy Ment Health. 2013;40(3):211-23.

16. Reberte LM, Hoga LAK, Gomes ALZ. O processo de construção de material educativo para a promoção da saúde da gestante. Rev Latinoam Enferm [Internet]. 2012 [acesso em 2017 Fev 16]; 20(1):1-8. Disponível em: http://www.scielo.br/ $\mathrm{pdf} / \mathrm{rlae} / \mathrm{v} 20 \mathrm{n} 1 / \mathrm{pt} \_14$

17. Defilipo ÉC, Frônio JS, Teixeira MTB, Leite ICG, Bastos RR, Vieira MT, et al. Oportunidades do ambiente domiciliar para o desenvolvimento motor. Rev Saúde Pública. 2012;46(4):633-41.

18. Silva GRF, Cardoso MVLML. Percepção de mães sobre um manual educativo sobre estimulação visual da criança. Rev Eletrônica Enferm [Internet]. 2009 [acesso em 2017 Fev 16]; 11(4):847-57. Disponível em: https://www.revistas.ufg.br/ fen/article/view/3324

\section{Endereço para correspondência:}

Hitallo Lima da Silva

Centro Universitário Estácio do Ceará

Rua Eliseu Uchôa Beco, 600

Bairro: Água Fria

CEP: 60810-270 - Fortaleza - CE - Brasil

E-mail: hitalols@hotmail.com 